Supporting Figures

\title{
Mapping the molecular architecture required for lipid-binding pockets using a subset of established and orphan GPCRs.
}

Shanthi Nagarajan ${ }^{1,2,5}$, Zu Yuan Qian ${ }^{1,3}$, Parthiban Marimuthu ${ }^{4}$, Nabil J. Alkayed ${ }^{1,3}$, Sanjiv Kaul ${ }^{1}$ and Anthony P. Barnes ${ }^{1,3}$

${ }^{1}$ The Knight Cardiovascular Institute, ${ }^{2}$ Medicinal Chemistry Core, ${ }^{3}$ Department of Anesthesiology \& Perioperative Medicine, Oregon Health Science University

Portland, OR 97239, USA. ${ }^{4}$ Pharmaceutical Science Laboratory and Structural Bioinformatics

Laboratory, Faculty of Science and Engineering, Åbo Akademi University, FI-20520 Turku,

Finland

${ }^{5}$ Current affiliation: Discovery Chemistry and Research Technologies, Lilly Research

Laboratories, Eli Lilly and Company, Lilly Corporate Center, Indianapolis, IN 46225, USA

Contact email: barnesan@ohsu.edu 


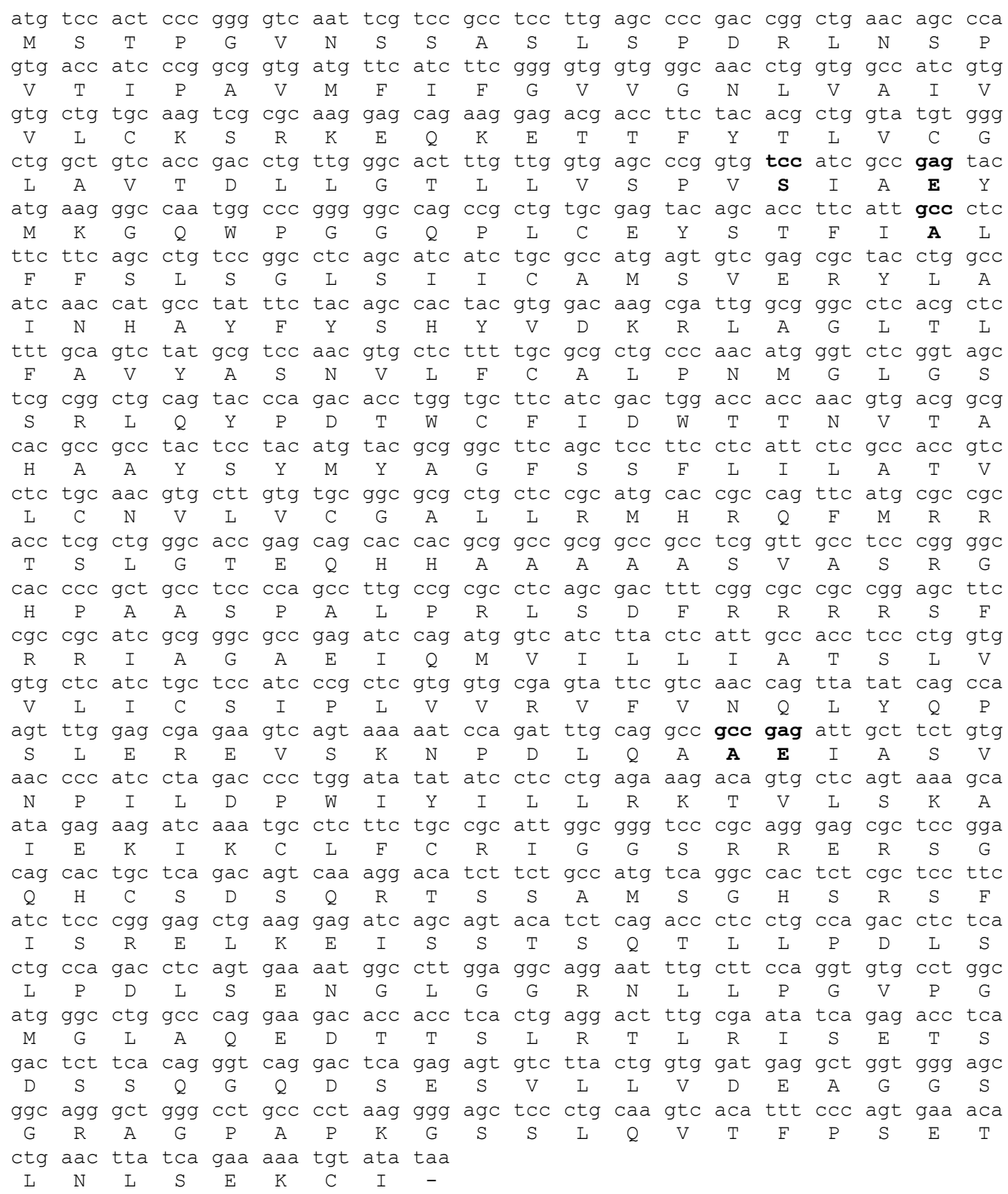

Figure S1A: Nucleotide sequence and protein translation of the PTGER 4 mutant. Altered nucleotides and amino acids are indicated in bold. 


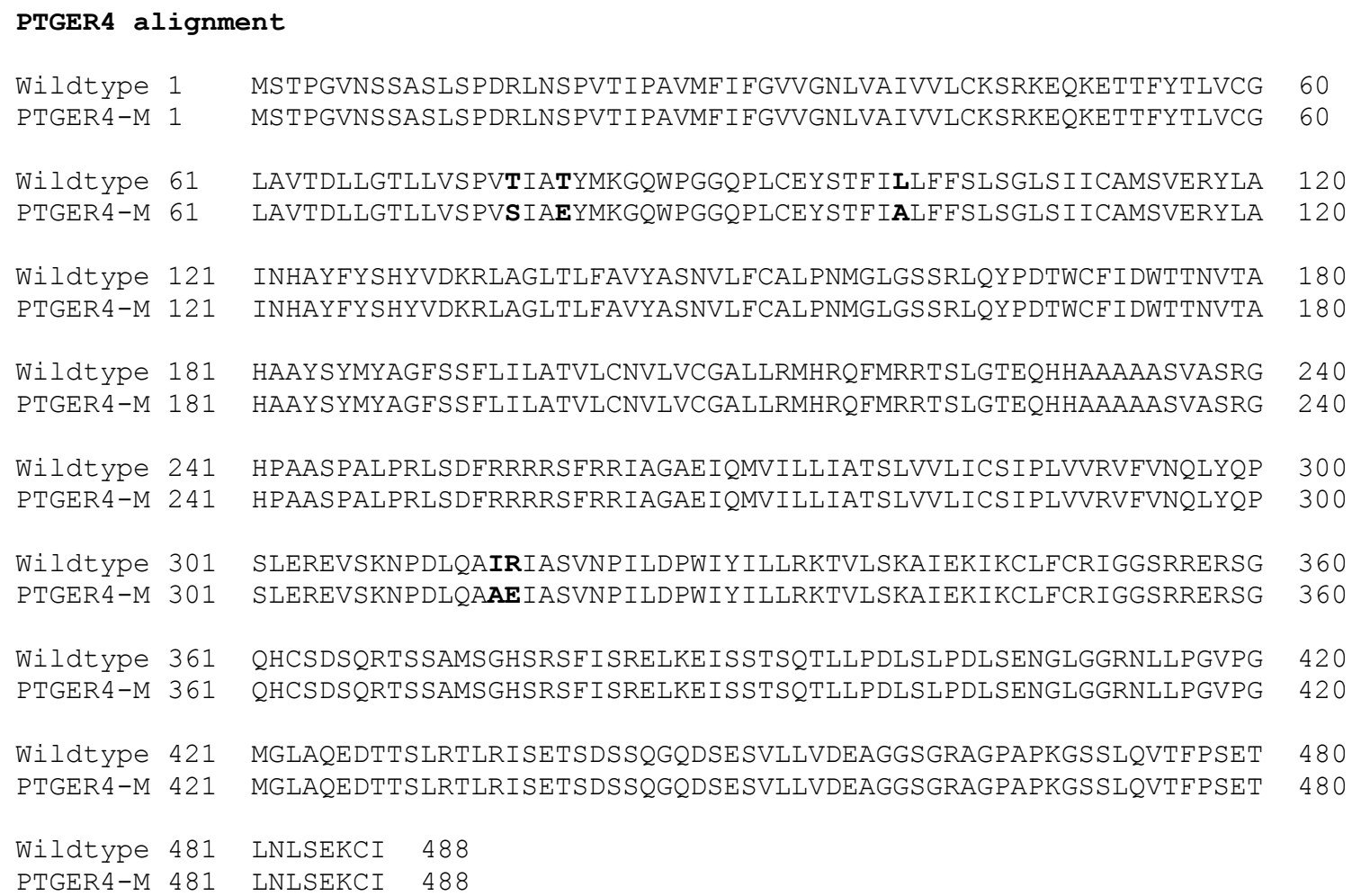

Figure S1B: Protein sequence alignment between the wildtype and mutant PTGER 4 . Altered amino acids and their wildtype counterparts are indicated in bold. 
CCR1 Mutant

atg gaa act cca aac acc aca gag gac tat gac acg acc aca gag ttt gac tat ggg gat $\begin{array}{llllllllllllllllllllllllllllll}M & E & T & P & N & T & T & E & D & Y & D & T & T & T & E & F & D & Y & G & D\end{array}$ gca act ctg tgc cag aag gtg aac gag agg gcc ttt atg gcc caa ctg gtg ccc cct ctg $\begin{array}{llllllllllllllllllllllllllllll}A & \mathrm{~T} & \mathrm{~L} & \mathrm{C} & \mathrm{Q} & \mathrm{K} & \mathrm{V} & \mathrm{N} & \mathrm{E} & \mathrm{R} & \mathrm{A} & \mathrm{F} & \mathbf{M} & \mathrm{A} & \mathrm{Q} & \mathrm{L} & \mathrm{V} & \mathrm{P} & \mathrm{P} & \mathrm{L}\end{array}$ tac tcc ttg gta ttt gtc att ggc ctg gtt gga aac atc ctg gtg gtc ctg gtc ctt gtg

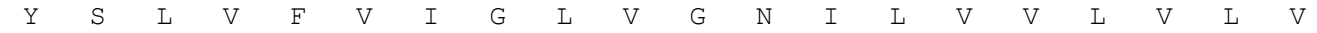
caa tac aag agg cta aaa aac atg acc agc atc tac ctc ctg aac ctg gec att tct gac

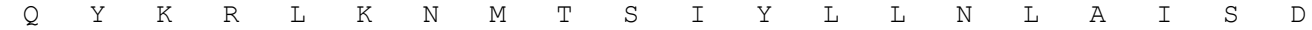
ctg ctc ttc ctg ttc acg ctt ccc ttc tgg atc gac tac gtg cgg aag gat gac tgg gtt $\begin{array}{lllllllllllllllllllllllllllll}\mathrm{L} & \mathrm{L} & \mathrm{F} & \mathrm{L} & \mathrm{F} & \mathrm{T} & \mathrm{L} & \mathrm{P} & \mathrm{F} & \mathrm{W} & \mathrm{I} & \mathrm{D} & \mathrm{Y} & \mathbf{V} & \mathbf{R} & \mathrm{K} & \mathrm{D} & \mathrm{D} & \mathrm{W} & \mathrm{V}\end{array}$ ttt ggt gat gcc atg tgt aag atc ctc tct ggg tt tat tac aca ggc ttg tac agc gag

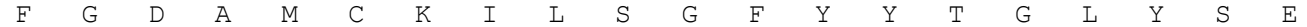
atc ttt ttc atc atc ctg ctg acg att gac agg tac ctg gcc atc gtc cac gcc gtg ttt $\begin{array}{llllllllllllllllllllllll}I & F & F & I & I & L & L & T & I & D & R & Y & L & A & I & V & H & A & V & F\end{array}$ gec ttg cgg gea cgg acc gtc act ttt ggt gtc atc acc agc atc atc att tgg gcc ctg

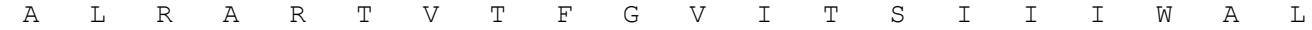
gcc atc ttg get tcc atg cca ggc tta tac ttt tcc aag acc caa tgg gaa ttc act cac $\begin{array}{lllllllllllllllllllll}\text { A } & \text { I } & \text { L } & \text { A } & \text { S } & \text { M } & \text { P } & \text { G } & \text { L } & \text { Y } & \text { F } & \text { S } & \text { K } & \text { T } & Q & W & E & F & T & H\end{array}$ cac acc tgc agc ctt cac tac cet cac gaa agc gtg cga gag tgg cgg ctg ttt cac acc

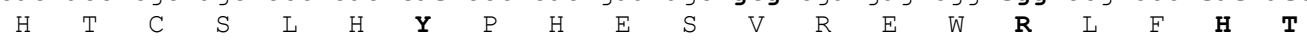
ctg aaa atg aac ctc ttt ggg ctg gta ttg cct ttg ttg gtc atg atc atc tgc tac aca

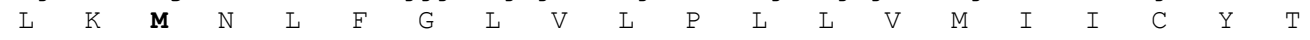
ggg att ata aag att ctg cta aga cga cca aat gag aag aaa tcc aaa gct gtc cgt ttg $\begin{array}{llllllllllllllllllllllll}G & I & I & K & I & L & L & R & R & P & N & E & K & K & S & K & A & V & R & L\end{array}$ att ttt gtc atc atg atc atc ttt ttt ctc ttt tgg acc ccc tac aat ttg act ata ctt

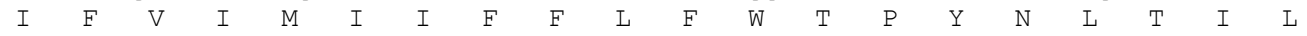
att tct tcc ttc caa gac ttc ctg ttc acc cat gag tgt gag cag agc aga cat ttg gac $\begin{array}{llllllllllllllllllllllllll}I & S & S & F & Q & D & F & L & F & T & H & E & C & E & Q & S & R & H & L & D\end{array}$ ctg get atg ctg gtg acg gag gtg atc gcc tac acg cac tgc tgt gtc aac cca gtg atc $\begin{array}{lllllllllllllllllllllllll}\mathrm{L} & \mathrm{A} & \mathbf{M} & \mathrm{L} & \mathrm{V} & \mathrm{T} & \mathrm{E} & \mathrm{V} & \mathrm{I} & \mathrm{A} & \mathrm{Y} & \mathrm{T} & \mathrm{H} & \mathrm{C} & \mathrm{C} & \mathrm{V} & \mathrm{N} & \mathrm{P} & \mathrm{V} & \mathrm{I}\end{array}$ tac gcc ttc gtt ggt gag agg ttc cgg aag tac ctg cgg cag ttg ttc cac agg cgt gtg $\begin{array}{llllllllllllllllllll}\mathrm{Y} & \mathrm{A} & \mathrm{F} & \mathrm{V} & \mathrm{G} & \mathrm{E} & \mathrm{R} & \mathrm{F} & \mathrm{R} & \mathrm{K} & \mathrm{Y} & \mathrm{L} & \mathrm{R} & \mathrm{Q} & \mathrm{L} & \mathrm{F} & \mathrm{H} & \mathrm{R} & \mathrm{R} & \mathrm{V}\end{array}$ get gtg cac ctg gtt aaa tgg ctc ccc ttc ctc tcc gtg gac agg ctg gag agg gtc agc

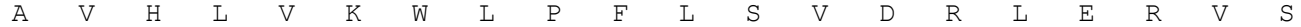
tcc aca tct ccc tcc aca ggg gag cat gaa ctc tet get ggg ttc tga

$\begin{array}{lllllllllllllllllll}S & T & S & P & S & T & G & E & H & E & L & S & A & G & F & -\end{array}$

Figure S2A: Nucleotide sequence and protein translation of the CCR1 mutant. Altered nucleotides and amino acids are indicated in bold. 


\begin{tabular}{|c|c|c|c|}
\hline Wildtype & 1 & METPNTTEDYDTTTEFDYGDATPCQKVNERAFGAQLLPPLYSLVFVIGLVGNILVVLVLV & 60 \\
\hline CCR1M & 1 & METPNTTEDYDTTTEFDYGDATLCQKVNERAFMAQLVPPLYSLVFVIGLVGNILVVLVLV & 60 \\
\hline Wildtype & 61 & QYKRLKNMTSIYLLNLAISDLLFLFTLPFWIDYKLKDDWVFGDAMCKILSGFYYTGLYSE & 20 \\
\hline CCR $1 \mathrm{M}$ & 61 & QYKRLKNMTSIYLLNLAISDLLFLFTLPFWIDYVRKDDWVFGDAMCKI LSGFYYTGLYSE & 120 \\
\hline Wildtype & 121 & IFFIILLTIDRYLAIVHAVFALRARTVTFGVITSIIIWALAILASMPGLYFSKTQWEFTH & 0 \\
\hline CCR $1 \mathrm{M}$ & 121 & IFFIILLTIDRYLAIVHAVFALRARTVTFGVITSIIIWALAILASMPGLYFSKTQWEFTH & 180 \\
\hline Wildtype & 181 & HTCSLHFPHESLREWKLFQALKLNLFGLVLPLLVMI ICYTGI IKILLRRPNEKKSKAVRL & \\
\hline CCR1M & 181 & HTCSLHYPHESVREWRLFHTLKMNLFGLVLPLLVMI ICYTGI IKILLRRPNEKKSKAVRL & 240 \\
\hline Wildtype & 241 & IFVIMIIFFLFWTPYNLTILISVEQDFLFTHECEQSRHLDLAVQVTEVIAYTHCCVNPVI & 0 \\
\hline CCR1M & 241 & IFVIMIIFFLFWTPYNLTILISSFQDFLFTHECEQSRHLDLAMLVTEVIAYTHCCVNPVI & 300 \\
\hline Wildtype & 301 & YAFVGERFRKYLRQLFHRRVAVHLVKWLPFLSVDRLERVSSTSPSTGEHELSAGF & \\
\hline CCR1M & 301 & YAFVGERFRKYLRQLFHRRVAVHLVKWLPFLSVDRLERVSSTSPSTGEHELSAGF & \\
\hline
\end{tabular}

Figure S2B: Protein sequence alignment between the wildtype and mutant CCR1. Altered amino acids and their wildtype counterparts are indicated in bold. 


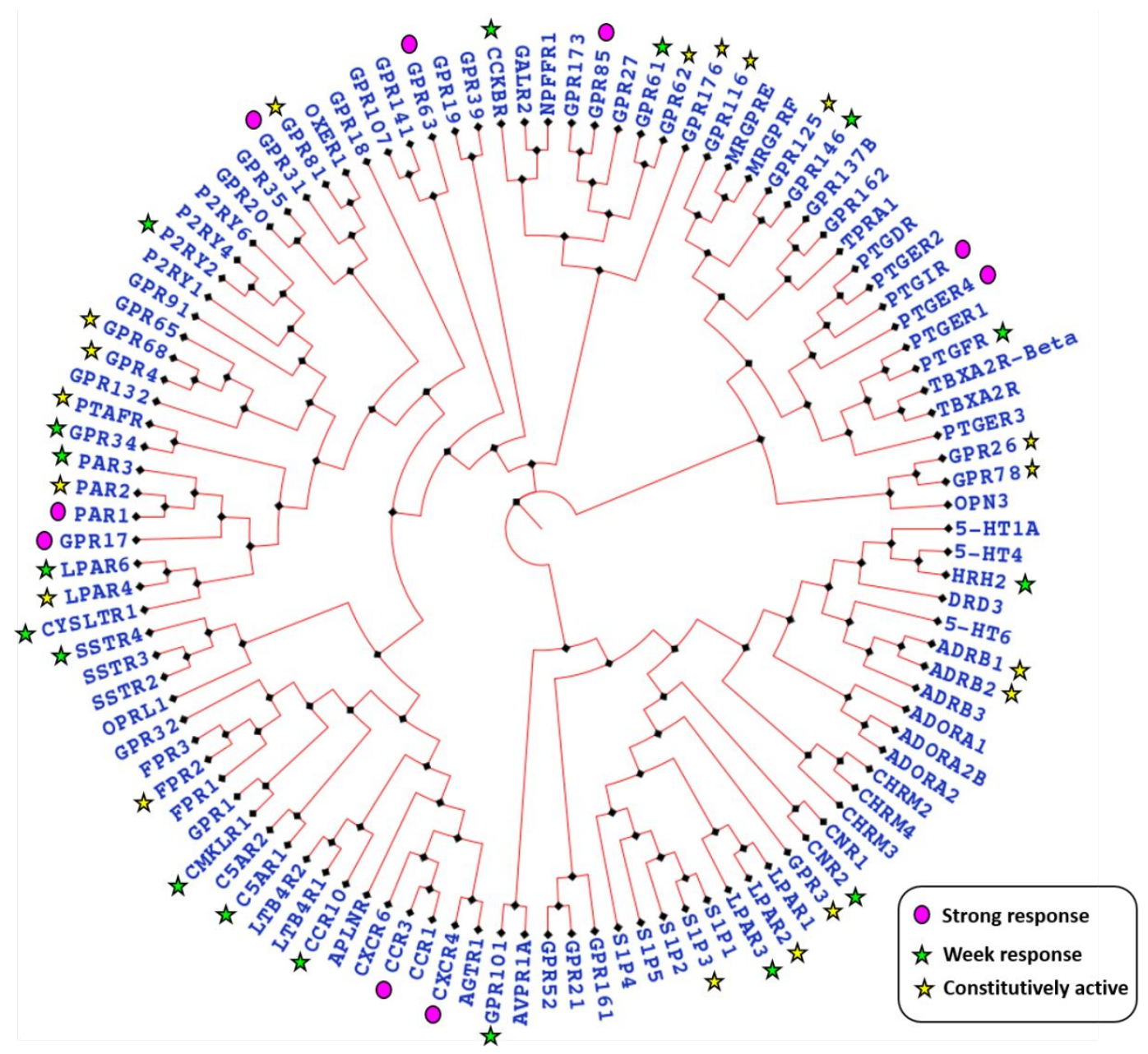

Figure S3: Dendrogram of receptors screened using $\mathrm{pERK}_{1 / 2}$ assay. Receptors response to $1 \mu \mathrm{M}$ 14,15-EET and constitutive activity is labeled. Unlabeled receptors indicate no response. 


\section{Supplemental Table}

\begin{tabular}{|c|c|c|c|c|c|}
\hline No. & Target & Template & PDB & $\begin{array}{c}\text { Similarity for } \\
\text { aligned } \\
\text { regions }\end{array}$ & $\begin{array}{l}\text { ID for aligned } \\
\text { region }\end{array}$ \\
\hline 1 & $\mathrm{PTGER}_{4}$ & 5-HT2B, Ergotamine (2.7 A) & 4IB4 & 43.8 & 20.6 \\
\hline 2 & $\mathrm{CCR}_{3}$ & $\mathrm{CCR}_{5}$, Maraviroc $(2.71 \mathrm{~A})$ & $4 \mathrm{MBS}$ & 80 & 55.6 \\
\hline 3 & GPR17 & P2Y1R, BPTU (2.2 A) & 4XNV & 50.1 & 26.5 \\
\hline 4 & GPR85 & $\begin{array}{l}\text { orexin receptor } 1 \text {, suvorexant } \\
(2.75 \mathrm{~A})\end{array}$ & 4ZJ8 & 48.9 & 20.3 \\
\hline 5 & $\mathrm{CXCR}_{4}$ & $\mathrm{CXCR}_{4}, \operatorname{ITD}(2.5 \mathrm{~A})$ & $3 \mathrm{ODU}$ & N.D. & N.D. \\
\hline 6 & PTGIR & $\begin{array}{l}\text { kappa opioid receptor, JDTic } \\
(2.9 \mathrm{~A})\end{array}$ & $4 \mathrm{DJH}$ & 41.1 & 17.7 \\
\hline 7 & GPR31 & $\begin{array}{l}\text { orexin receptor } 2 \text {, Suvorexant } \\
(2.5 \mathrm{~A})\end{array}$ & $4 \mathrm{~S} 0 \mathrm{~V}$ & 47.7 & 20.6 \\
\hline 8 & $\mathrm{PAR}_{1}$ & $\mathrm{PAR}_{1}$, vorapaxar $(2.2 \mathrm{~A})$ & 3VW7 & N.D. & N.D. \\
\hline 9 & GPR63 & $\begin{array}{l}\text { 5-HT1B, Dihydroergotamine } \\
(2.8 \mathrm{~A})\end{array}$ & 4IAQ & 51.9 & 27.4 \\
\hline
\end{tabular}

N.D.: not determined as the crystal structures was available.

Table S1: Template structures used to generate a homology model and identify similarity with target sequence. 